\title{
Marcadores de fase aguda en niños y adolescentes obesos con trastornos metabólicos
}

\author{
Acute phase markers in obese children and adolescents with metabolic disorders
}

\author{
Dra. Idalia Cura-Esquivel ${ }^{a}$, Dra. Paula Cordero-Pérez ${ }^{b}$,Dra. Liliana Torres-González $z^{b}$ \\ Dra. Linda E. Muñoz-Espinosa $a^{b}$
}

\section{RESUMEN}

Introducción. La obesidad es un problema de salud pública mundial y la enfermedad crónica no transmisible más frecuente. Se asocia con la elevación de proteínas inflamatorias de fase aguda y citocinas proinflamatorias.

Objetivo. Evaluar los niveles de proteínas de fase aguda en niños y adolescentes obesos con esteatosis hepática y síndrome agudo metabólico.

Metodología. Se incluyeron 45 niños con índice de masa corporal $\geq$ percentil 95, de edades entre 5,0 y 15,5 años. Se determinaron reactantes de fase aguda: proteína $C$ reactiva, haptoglobina, $\alpha-2$ macroglobulina y apolipoproteína A-1, y se realizó una ecografía para evaluar la esteatosis hepática.

Resultados. Todos los pacientes mostraron una elevación de proteína $C$ reactiva. Los pacientes con síndrome metabólico también tuvieron un incremento en la apolipoproteína A-1 y la haptoglobina. Los pacientes con esteatosis hepática tuvieron un aumento significativo en la $\alpha-2$ macroglobulina además de la protenína $C$ reactiva.

Palabras clave: obesidad, proteina $C$ reactiva, haptoglobinas, $\alpha-2$ macroglobulinas, apolipoproteína A-1.

http: / / dx.doi.org/10.5546/ aap.2018.275

Texto completo en inglés:

http:/ / dx.doi.org/10.5546/ aap.2018.eng.275

Cómo citar: Cura-Esquivel I, Cordero-Pérez P, Torres-González L, Muñoz-Espinosa LE. Marcadores de fase aguda en niños y adolescentes obesos con trastornos metabólicos. Arch Argent Pediatr 2018;116(4):275279.

a. Departamento de Pediatría, Hospital Universitario "Dr. José E. González".

b. Unidad de Hígado, Hospital Universitario "Dr. José E. González".

Universidad Autónoma de Nuevo León, México.

Correspondencia:

Dra. Idalia Cura-Esquivel: idaliaaracely2008@hotmail.com

Financiamiento: Este estudio recibió apoyo financiero del Programa de Apoyo a la Investigación Científica y Tecnológica (PAICYT) de la Universidad Autónoma de Nuevo León.

Conflicto de intereses: Ninguno que declarar.

Recibido: 9-6-2017

Aceptado: 7-12-2017

\section{INTRODUCCIÓN}

La obesidad se define como una acumulación excesiva de grasa corporal ocasionada por una alta ingesta de energía superpuesta a un bajo gasto. Constituye la enfermedad crónica no transmisible más frecuente tanto en adultos como en niños. ${ }^{1,2}$ En México, la prevalencia de sobrepeso y obesidad en escolares es de 33,2\%, según la última Encuesta Nacional de Salud y Nutrición (ENSANUT 2016). ${ }^{3}$ La obesidad es regulada por una serie de adipocinas, entre las cuales se incluyen la leptina, la adiponectina, la haptoglobina y el amiloide sérico. Estos mediadores producidos en el tejido adiposo inducen un estado de inflamación sistémica de bajo grado y este proceso es un mecanismo que relaciona la obesidad con un espectro de condiciones patológicas asociadas. ${ }^{4}$ Múltiples estudios demuestran la asociación entre el índice de masa corporal (IMC) y la obesidad visceral y sus complicaciones, como el síndrome metabólico (SM), con niveles elevados de mediadores de inflamación no específicos, como la proteína $C$ reactiva $(\mathrm{PCR})$, proteínas inflamatorias de fase aguda y otras citocinas proinflamatorias. ${ }^{4,5}$

El objetivo del presente estudio fue evaluar los niveles de proteínas de fase aguda en niños y adolescentes obesos con esteatosis hepática y SM.

\section{POBLACIÓN Y MÉTODOS}

Estudio observacional, longitudinal, analítico y prospectivo entre enero de 2009 y diciembre de 2013. Se incluyeron pacientes de entre 5 y 15,5 años de edad, tratados en la consulta de Pediatría del Hospital Universitario “Dr. José Eleuterio González”, Universidad Autónoma de Nuevo León (UANL), con IMC $\geq$ percentil 95 de acuerdo con las gráficas para la edad y el sexo del Centro de Control y Prevención de Enfermedades (Centers for Disease Control and Prevention; CDC, por sus siglas en inglés). El IMC fue calculado sobre la base de la fórmula peso $(\mathrm{kg}) /$ talla $\left(\mathrm{m}^{2}\right)$. Se realizó una evaluación clínica y antropométrica, que incluyó peso, talla y circunferencia de cintura (CC), además de una 
evaluación por ultrasonido abdominal, para definir la presencia de esteatosis hepática. El grado de esteatosis hepática se clasificó de 0 (sin esteatosis) a 3 (esteatosis grave). Se evaluó la presencia de SM de acuerdo con los criterios de la Federación Internacional de Diabetes (FID), que establecían presencia de obesidad central más la existencia de 2 o más datos clínicos: triglicéridos $\geq 150 \mathrm{mg} /$ dl, lipoproteínas de alta densidad (High density lipoprotein; HDL, por sus siglas en inglés) $<40 \mathrm{mg} / \mathrm{dl}$ en hombres y $<50 \mathrm{mg} / \mathrm{dl}$ en mujeres, hipertensión arterial (tensión arterial $-\mathrm{T} / \mathrm{A}->$ percentil 97), glucosa $>100$ carga oral de glucosa o diabetes mellitus (DM) tipo 2, cintura $\geq$ percentil $90 .{ }^{6}$ Los pacientes con diagnóstico previo de enfermedad hepática aguda o crónica, alteraciones endocrinológicas, genéticas, renales o que rechazaran participar en el estudio fueron excluidos.

Se recolectaron $8 \mathrm{ml}$ de sangre $(5 \mathrm{ml}$ para suero y $3 \mathrm{ml}$ para sangre con EDTA) para la determinación de las proteínas de fase aguda y las pruebas de funcionamiento hepático. Se realizaron las mediciones en suero de PCR, haptoglobina, $\alpha-2$ macroglobulina $(\alpha-2 M G)$ y apolipoproteína A-1 (APO A-1).

El análisis descriptivo para las variables continuas fue expresado como valores medios \pm desviación estándar (DE). Las variables categóricas fueron expresadas como frecuencias y proporciones. La relación entre las pruebas bioquímicas y los marcadores inflamatorios agudos se analizó mediante la prueba de correlación de Spearman para los datos no paramétricos y por el método de Pearson para los datos paramétricos. Para ello, se utilizaron los programas estadísticos SPSS versión 16.0. Se consideró estadísticamente significativo el valor de $\mathrm{p}<0,05$.

\section{Aspectos éticos}

Este estudio fue aprobado por el Comité de Ética de nuestra Institución (PE08-008). Todos los pacientes contaron con su asentimiento y con la carta de consentimiento informado firmada por los padres.

\section{RESULTADOS}

Se incluyeron 45 pacientes, $19(46 \%)$ mujeres y $26(57,8 \%)$ varones. La edad media del grupo fue de $11 \pm 2,2$ años (de 5 a 15 años). El IMC fue de $27,07 \pm 5,9 \mathrm{~kg} / \mathrm{m}^{2}$ de superficie corporal (SC) (de 22,4 a $37,8 \mathrm{~kg} / \mathrm{m}^{2}$ de SC). Todos los pacientes tuvieron diagnóstico de obesidad de acuerdo con el percentil de IMC. La CC fue de $90 \pm 17 \mathrm{~cm}$ con un índice cintura/cadera de 0,85 $\pm 0,18 \mathrm{~cm}$.

\section{Reactantes de fase aguda}

Solo la PCR $(4,65 \pm 5,5 \mathrm{mg} / \mathrm{dl} \mathrm{vs}$. $0,25 \pm 0,33 \mathrm{mg} / \mathrm{dl}, \mathrm{p}<0,001)$ tuvo niveles elevados significativamente en todos los pacientes. Al evaluar los niveles por sexo, las mujeres tuvieron más elevados que los varones $(5,72 \pm 7,5$ vs. $3,34 \pm 3,77 \mathrm{mg} / \mathrm{dl}, \mathrm{p}=0,02)$, contrario a lo que ocurrió con la $\alpha-2 \mathrm{MG}$, la cual fue más elevada en los hombres $(3,05 \pm 0,60$ vs. $2,74 \pm 0,43 \mathrm{mg} / \mathrm{dl}, \mathrm{p}<0,05)$. Al correlacionar los parámetros antropométricos con los bioquímicos y las proteínas de fase aguda, se observó una correlación positiva de la PCR con el IMC $(\mathrm{r}=0,34, \mathrm{p}<0,01)$, con la CC $(\mathrm{r}=0,76, \mathrm{p}<0,001)$, con la gammaglutamil transferasa (GGT) $(r=$ $0,38, \mathrm{p}<0,04)$, con la $\alpha-2 \mathrm{MG}(\mathrm{r}=0,29, \mathrm{p}<0,04)$ y con la APO A-1 $(\mathrm{r}=0,32, \mathrm{p}<0,03)$ (Tabla 1$)$.

\section{Obesidad y síndrome metabólico}

De acuerdo con los criterios de la FID, $24(53 \%)$ pacientes, 8 mujeres y 16 hombres, tuvieron diagnóstico de SM. Los niveles de PCR fueron más elevados en los pacientes con SM $(4,05 \pm 5,1$ vs. $2,15 \pm 4,7 \mathrm{mg} / \mathrm{dl}, \mathrm{p}<0,001)$. Se observó un incremento gradual con el aumento de número de componentes del SM. La media de PCR para aquellos con $0,1,2,3$ y 4 datos de SM fue de 2,6, 2,52, 2,86, 6,04 y 16,5 mg/ $\mathrm{dl}$, respectivamente. La APO A-1 (1,14 $\pm 0,33$ vs. $0,50 \pm 0,56 \mathrm{mg} / \mathrm{dl}, \mathrm{p}<0,001)$, al igual que la haptoglobina $(1,46 \pm 0,26$ vs. $1,30 \pm 0,27 \mathrm{mg} /$ dl, p < 0,001), fueron más elevadas, con significancia estadística, mientras que la $\alpha-2 \mathrm{MG}$ fue ligeramente mayor en el grupo con $\mathrm{SM}$, sin diferencia significativa $(\mathrm{p}=0,08)$ (Figura 1).

\section{Obesidad y esteatosis hepática}

Se diagnosticó esteatosis hepática por ultrasonido en $21(46,6 \%)$ pacientes, 13 esteatosis leve, 6 esteatosis moderadas y 2 esteatosis graves. Los niveles de PCR $(5,2 \pm 6,6 \mathrm{mg} /$ dl vs. $1,9 \pm 3,6 \mathrm{mg} / \mathrm{dl}, \mathrm{p}<0,001)$ y $\alpha-2 \mathrm{MG}$ $(3,12 \pm 0,68$ vs. $2,75 \pm 0,35 \mathrm{mg} / \mathrm{dl}, \mathrm{p}=0,03)$ fueron significativamente más elevados en los pacientes con esteatosis hepática, mientras que la haptoglobina (1,44 $\pm 0,27$ vs. $1,38 \pm 0,23 \mathrm{mg} / \mathrm{dl}, \mathrm{p}$ $=0,82)$ y la APO A- $1(1,15 \pm 0,29$ vs. $1,05 \pm 0,537$ $\mathrm{mg} / \mathrm{dl}, \mathrm{p}=0,09)$ no mostraron diferencia estadísticamente significativa (Figura 2).

\section{DISCUSIÓN}

La PCR y otros reactantes de fase aguda han sido involucrados en el diagnóstico del estado inflamatorio que se describe en pacientes con 
obesidad. La $\alpha-2 \mathrm{MG}$ es una proteína de origen hepático que, en los pacientes con hígado graso no alcohólico, se ha asociado con el proceso de fibrogénesis.

La APO A-1, también sintetizada en el hígado, constituye el principal componente de las lipoproteínas de alta densidad y juega un rol en la homeostasis del colesterol y disminuye con la progresión de fibrosis y cirrosis hepática, mientras que la haptoglobina, también de síntesis hepática, disminuye su concentración durante la fibrogénesis. ${ }^{78}$ Nuestros resultados evidencian una correlación positiva entre el IMC elevado con los reactantes de fase aguda.

El ultrasonido hepático es un estudio de imagen seguro, bien tolerado, económico,

TABLA 1. Análisis de correlación entre las variables antropométricas y bioquímicas

\begin{tabular}{|c|c|c|c|c|c|c|c|c|}
\hline \\
\hline \multicolumn{9}{|l|}{$\begin{array}{l}N=45 \\
\text { Variable }\end{array}$} \\
\hline Triglicéridos & $0,08 / 0,57$ & & & & & & & \\
\hline CT & $-0,13 / 0,37$ & $0,58 / 0,70$ & & & & & & \\
\hline ALT & $-0,09 / 0,95$ & $-0,23 / 0,87$ & $0,98 / 0,52$ & & & & & \\
\hline AST & $-0,22 / 0,14$ & $-0,16 / 0,29$ & $0,99 / 0,51$ & $0,68 / 0,01$ & & & & \\
\hline FA & $-0,15 / 0,31$ & $0,61 / 0,69$ & $0,14 / 0,33$ & $0,24 / 0,10$ & $0,11 / 0,45$ & & & \\
\hline GGT & $0,78 / 0,61$ & $0,98 / 0,52$ & $0,82 / 0,59$ & $0,51 / 0,01$ & $0,44 / 0,02$ & & & \\
\hline Glucosa & $0,28 / 0,05$ & $0,45 / 0,77$ & $0,29 / 0,04$ & $0,11 / 0,23$ & $0,21 / 0,15$ & $0,21 / 0,15$ & & \\
\hline PCR & $0,34 / 0,01$ & $0,53 / 0,72$ & $-0,45 / 0,76$ & $0,06 / 0,69$ & $0,38 / 0,80$ & $0,38 / 0,04$ & $0,13 / 0,38$ & \\
\hline$\alpha-2 \mathrm{MG}$ & $0,06 / 0,97$ & $0,06 / 0,68$ & $0,29 / 0,05$ & $-0,02 / 0,87$ & $-0,12 / 0,40$ & $0,04 / 0,97$ & $0,09 / 0,55$ & $-0,29 / 0,04$ \\
\hline APO A-1 & $0,22 / 0,13$ & $-0,20 / 0,05$ & $0,20 / 0,17$ & $0,13 / 0,39$ & $0,10 / 0,48$ & $0,08 / 0,58$ & $0,30 / 0,03$ & $0,32 / 0,03$ \\
\hline Haptoglobina & $-0,20 / 0,18$ & $0,02 / 0,89$ & $0,56 / 0,01$ & $0,25 / 0,09$ & $0,34 / 0,02$ & $0,20 / 0,16$ & $-0,09 / 0,52$ & $0,12 / 0,40$ \\
\hline
\end{tabular}

IMC: índice de masa corporal; CT: colesterol total; ALT: alanina aminotransferasa; AST: aspartato aminotransferasa; FA: fosfatasa alcalina; GGT: gammaglutamil transferasa; PCR: proteína $C$ reactiva; $\alpha-2 M G: \alpha-2$ macroglobulina; APO A-1: apolipoproteína A-1.

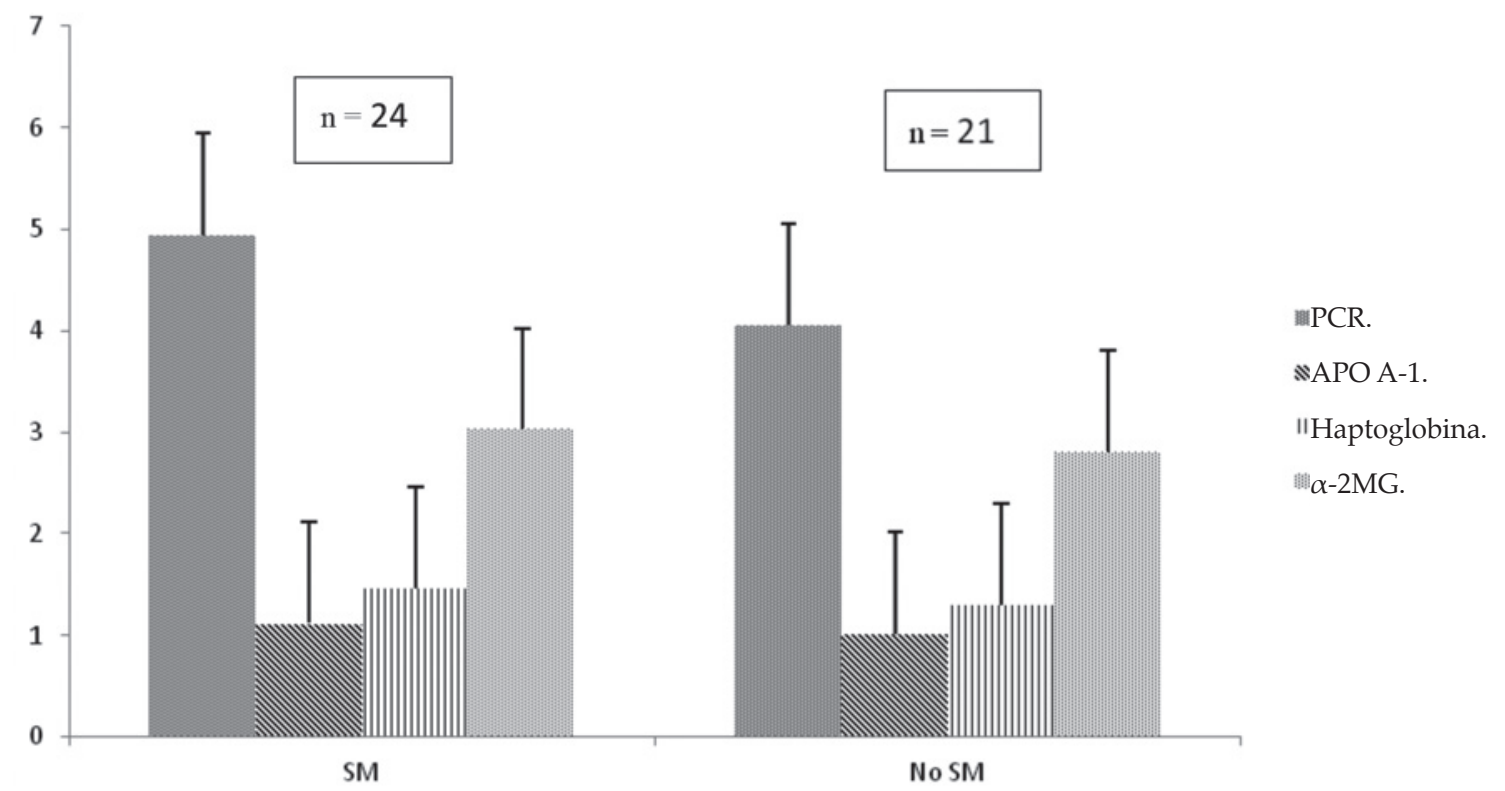

SM: síndrome metabólico; PCR: proteína C reactiva; APO A-1: apolipoproteína A-1; $\alpha$-2MG: $\alpha 2$ macroglobulinas. 
disponible y aceptado para el diagnóstico de hígado graso. Estudios recientes demuestran que es sensible para detectar un grado de esteatosis tan bajo como 10-20\%. ${ }^{9}$ El diagnóstico de esteatosis hepática se realizó por ultrasonido en 21 pacientes y fue, en la mayoría, una esteatosis leve y tuvo niveles de PCR y $\alpha$-2MG significativamente más elevados que en los pacientes sin esteatosis. Nuestros hallazgos son consistentes con lo observado en estudios previos, lo cual sugiere inflamación de bajo grado en el hígado.

Algunos autores, como Bogdanski et al., y Vallianou et al., evaluaron los reactantes de fase aguda (PCR, haptoglobina, $\alpha-2 M G$ ) en pacientes con SM y encontraron que estas proteínas estaban elevadas significativamente en pacientes con SM. Sugirieron que tanto la PCR como la haptoglobina podían ser marcadores de gravedad del SM. ${ }^{7,10}$ En este estudio de PCR, la APO A-1 y la haptoglobina estaban significativamente elevadas en los pacientes con SM, también asociado con un estado de inflamación crónico en coincidencia con otros padecimientos que se desarrollaron con esteatosis y fibrosis como se observó en la Hepatitis C. ${ }^{11}$.

En nuestro estudio, la PCR, junto con la APO A-1, se asociaron a obesidad y SM, mientras que el IMC y la PCR se asociaron fuertemente con el grado de esteatosis.
Las debilidades de nuestro estudio fueron, sobre todo, el número de pacientes y el amplio rango de edades, lo que limitó el análisis. Tampoco se consideró el estadio puberal, que podría haber marcado diferencias en los hallazgos, especialmente, si se tomaba en cuenta el efecto de los estrógenos sobre el control transcripcional, aclaramiento y regulación de algunas proteínas producidas por el hígado (citocinas), que incluían la PCR, que podría haber influido en los resultados.

En conclusión, todos los pacientes mostraron un incremento de PCR independientemente del IMC y del estado metabólico. Los pacientes con SM también presentaron elevación de la APO A-1 y de la haptoglobina, mientras que los pacientes con esteatosis hepática tuvieron además un aumento significativo de la $\alpha-2 \mathrm{MG}$.

\section{REFERENCIAS}

1. Güngör NK. Overweight and obesity in children and adolescents. J Clin Res Pediatr Endocrinol 2014;6(3):129-43.

2. Han JC, Lawlor DA, Kimm SY. Childhood obesity. Lancet 2010;375(9727):1737-48.

3. Gutiérrez JP, Rivera-Dommarco J, Shamah-Levy T, et al. Encuesta Nacional de Salud y Nutrición 2016. Resultados Nacionales. Cuernavaca: Instituto Nacional de Salud Pública; 2016.

4. Singer $\mathrm{K}$, Lumeng $\mathrm{CN}$. The initiation of metabolic inflammation in childhood obesity. J Clin Invest 2017;127(1):65-73.

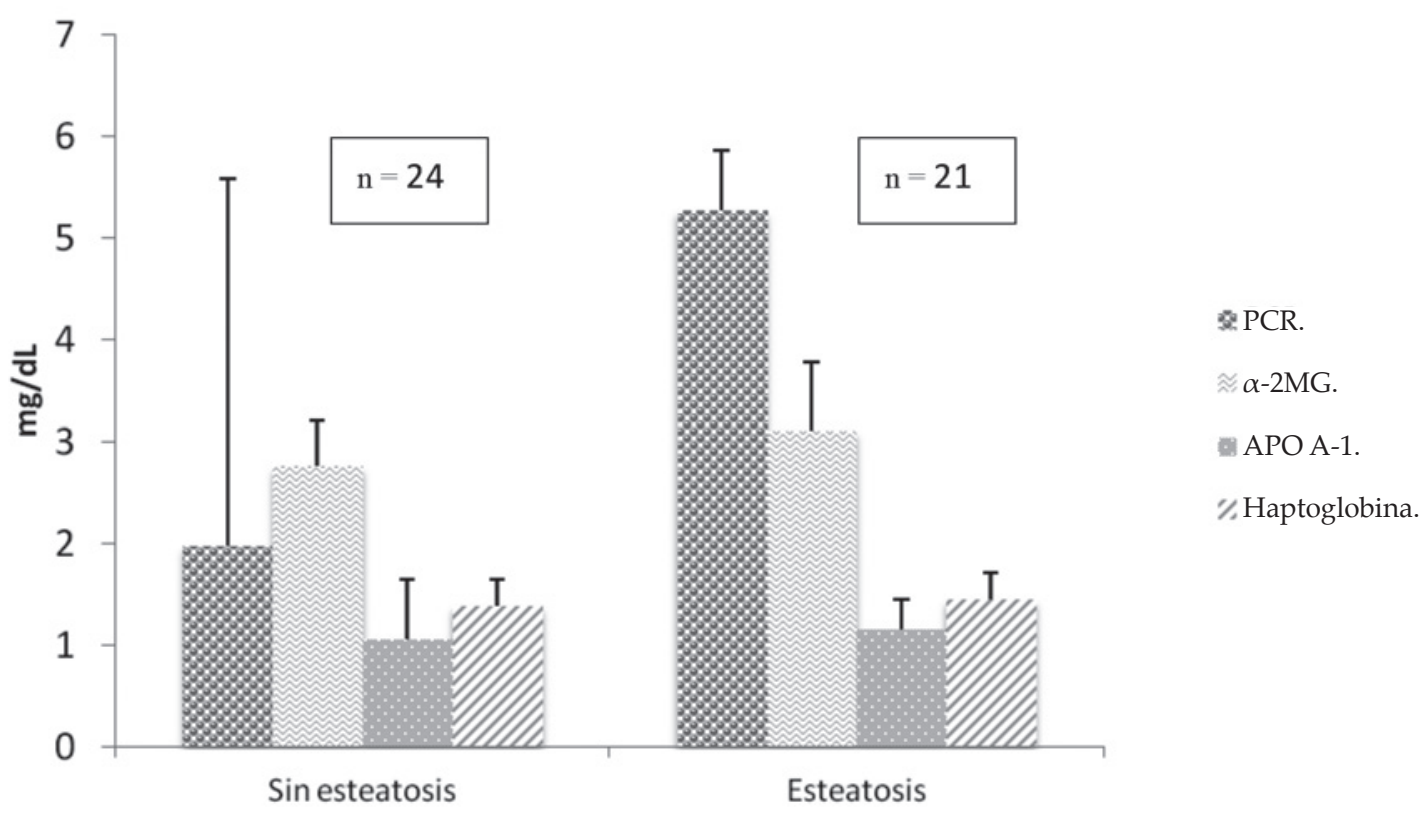

PCR: proteína C reactiva; APO A-1: apolipoproteína A-1; $\alpha$-2MG: $\alpha-2$ macroglobulina. 
5. Dedoussis GV, Kapiri A, Samara A, et al. Expression of inflammatory molecules and associations with BMI in children. Eur J Clin Invest 2010;40(5):388-92.

6. Alberti KG, Zimmet $P$, Shaw J, et al. The metabolic syndrome--a new worldwide definition. Lancet 2005;366(9491):1059-62.

7. Vallianou NG, Evangelopoulos AA, Panagiotakos DB, Georgiou AT, et al. Associations of acute-phase reactants with metabolic syndrome in middle-aged overweight or obese people. Med Sci Monit 2010;16(2):CR56-60.

8. Zimmermann E, Anty R, Tordjman J, et al. C-reactive protein levels in relation to various features of non- alcoholic fatty liver disease among obese patients. J Hepatol 2011;55(3):660-5.

9. Bril F, Ortiz-Lopez C, Lomonaco R, et al. Clinical value of liver ultrasound for the diagnosis of nonalcoholic fatty liver disease in overweight and obese patients. Liver Int 2015;35(9):2139-46.

10. BogdańskiP, Chyrek R, Pupek-MusialikD, etal.Evaluation of selected acute phase proteins in patients with metabolic syndrome. Pol Merkur Lekarski 2006;21(121):12-4.

11. ParkSH, Kim CH, Kim DJ, et al. Diagnostic value of multiple biomarker panel for prediction of significant fibrosis in chronic hepatitis C. Clin Biochem 2011;44(17-18):1396-9.

\title{
Caracterización de la consulta por intoxicación medicamentosa en los adolescentes en el Hospital Municipal de Bahía Blanca, Buenos Aires \\ Characterization of drug poisoning among adolescents seen at the municipal hospital of Bahía Blanca, Province of Buenos Aires, Argentina
}

\author{
Dr. Ignacio Buffone ${ }^{a}$, Dra. Mabel Dejter ${ }^{a}$, Dra. Eliana Fortunatti ${ }^{a}$ Dra. Florencia García Elliot ${ }^{a}$, \\ Dra. Camila Irazabal ${ }^{a}$, Dr. Rubén Marlia ${ }^{a}$, Dra. Daiana Mujica ${ }^{a}$, Dra. Marianela Parrou ${ }^{a}$ \\ Dra. Mariana Romano ${ }^{a}$, Dra. Gina Speciale y Dra. Agostina Werneke ${ }^{a}$
}

\section{RESUMEN}

Introducción. La consulta por intoxicación medicamentosa en los niños se da con frecuencia en los servicios de emergencias médicas; en la adolescencia, es cuando ocurren de forma intencional.

Objetivo. Caracterizar los patrones epidemiológicos de la intoxicación medicamentosa en adolescentes ingresados al Hospital Municipal de Bahía Blanca.

Material y método. Estudio descriptivo, basado en la revisión dehistorias clínicas de pacientes de 10 a 19 años que consultaron por intoxicación medicamentosa, entre los años 2012 y 2016. Las variables analizadas fueron edad, sexo, fármaco consumido, causa, manifestaciones clínicas y días de internación.

Resultados. Se recopilaron 72 pacientes. La edad media fue de 16 años, razón mujer-hombre de 2,5:1. Predominaron las intoxicaciones por ansiolíticos $(30 \%)$, seguidas de las

a. Servicio de Clínica Pediátrica, Hospital Municipal de Agudos “Dr. Leónidas Lucero", Bahía Blanca, provincia de Buenos Aires.

Correspondencia:

Dr. Ignacio Buffone: nacho_b89@hotmail.com

Financiamiento: Ninguno.

Conflicto de intereses: Ninguno que declarar.

Recibido: 16-7-2017

Aceptado: 21-12-2017 polimedicamentosas (25\%). El 95\% manifestaron intención suicida, de los cuales el 55\% ya tenían antecedentes.

Conclusión. La intoxicación medicamentosa ocurrió, predominantemente, en mujeres, y se destacó el gran porcentaje de adolescentes que presentaba episodios previos.

Palabras clave: adolescente, toxicidad de medicamentos.

http:/ / dx.doi.org/10.5546/ aap.2018.279

Texto completo en inglés:

http: / / dx.doi.org/10.5546/ aap.2018.eng.279

Cómo citar: Buffone I, Dejter M, Fortunatti E, et al. Caracterización de la consulta por intoxicación medicamentosa en los adolescentes en el Hospital Municipal de Bahía Blanca, Buenos Aires. Arch Argent Pediatr 2018;116(4):279-282.

\section{INTRODUCCIÓN}

La intoxicación medicamentosa en los adolescentes se ha transformado, en los últimos años, en un serio problema de salud pública, especialmente, en el área pediátrica, dado que son causantes de hasta un 10\% de todos los ingresos a la Unidad de Cuidados Intensivos, con tasas de mortalidad muy variables en los diferentes escenarios de atención. ${ }^{1}$

El espectro de sustancias implicadas en la intoxicación es amplio: los antidepresivos, los ansiolíticos y los antiinflamatorios no 